Bundesgesundheitsbl 2021 · 64:1093-1106 https://doi.org/10.1007/s00103-021-03394-x Eingegangen: 6. April 2021

Angenommen: 30. Juni 2021

Online publiziert: 10. August 2021

(c) Der/die Autor(en) 2021

Julia Schilling' - Kristin Tolksdorf' • Adine Marquis' • Mirko Faber' - Thomas Pfoch' Silke Buda ${ }^{1}$. Walter Haas' ${ }^{1}$ Ekkehard Schuler ${ }^{2}$. Doris Altmann' - Ulrike Grote' Michaela Diercke' $\cdot$ RKI COVID-19 Study Group

'Abteilung für Infektionsepidemiologie, Robert Koch-Institut, Berlin, Deutschland

${ }^{2} \mathrm{HELIOS}$ Kliniken GmbH, Berlin, Deutschland

\title{
Die verschiedenen Phasen der COVID-19-Pandemie in Deutschland: Eine deskriptive Analyse von Januar 2020 bis Februar 2021
}

\section{Einleitung}

Das neuartige Severe Acute Respiratory Syndrome Coronavirus 2 (SARS-CoV2) wurde Ende Dezember 2019 erstmals in Wuhan, China entdeckt und breitete sich innerhalb weniger Wochen weltweit aus. Am 27.01.2020 wurde der erste Fall mit einer SARS-CoV-2-Infektion in Deutschland diagnostiziert [1], bis zum Mai 2021 wurde Deutschland dann von insgesamt 3 COVID-19-Wellen erfasst. Der folgende Artikel hat das Ziel, Daten aus verschiedenen SurveillanceSystemen zu kombinieren, um einen umfassenden Überblick zum Verlauf der COVID-19-Pandemie bis zum Ende der zweiten Welle zu geben. Hierbei werden sowohl die geografische Ausbreitung als auch die Demografie der Fälle und die Krankheitsschwere im zeitlichen Verlauf und im Kontext der infektionshygienischen Maßnahmen betrachtet. Zudem werden die beiden ersten COVID-19Wellen in Deutschland verglichen.

Die Autorinnen J.Schilling und K. Tolksdorfteilen sich die Erstautorinnenschaft (alphabetisch geordnet).

\section{Methoden}

Das COVID-19-Geschehen in Deutschland wird retrospektiv in 4 Phasen dargestellt:

- Phase 0 (Auftreten sporadischer Fälle): Kalenderwoche $5 / 2020$ bis $9 / 2020$,

- Phase 1 (erste COVID-19-Welle): Kalenderwoche 10/2020 bis 20/2020,

- Phase 2 (Sommerplateau): Kalenderwoche $21 / 2020$ bis $39 / 2020$

(Phase 2a: $21 / 2020$ bis 30/2020 und

Phase 2b: 31/2020 bis 39/2020),

- Phase 3 (zweite COVID-19-Welle): Kalenderwoche $40 / 2020$ bis $8 / 2021$.

Die Abgrenzung der Phasen erfolgte anhand verschiedener epidemiologischer Parameter und wurde bereits an anderer Stelle ausführlich beschrieben [2]. Basierend auf diesem Vorgehen wurde das Ende der Phase 3 (zweite Welle) in Kalenderwoche (KW) 8/2021 festgelegt.

Es wurden die an das Robert KochInstitut (RKI) übermittelten Fälle gemäß Infektionsschutzgesetz (IfSG) entsprechend der RKI-Referenzdefinition [3] mit Datenstand 11.05.2021 betrachtet. In einer Analyse der Krankheitsschwere zur ersten COVID-19-Welle [4] hat sich herausgestellt, dass der Anteil intensivpflichtiger Fälle in den Meldedaten unterschätzt wird. Für ein vollständigeres Bild der kritischen Fälle wurden daher die ICD-10-code-basierten Daten zu hospitalisierten COVID-19-Fällen (COVID-SARI-Fälle) aus der Krankenhaus-Surveillance ICOSARI mit gleichem Datenstand herangezogen $[5,6]$.

COVID-19-Fälle aus dem Meldesystem gemäß Infektionsschutzgesetz

Der direkte oder indirekte Nachweis von SARS-CoV-2 sowie der Krankheitsverdacht, die Erkrankung und der Tod in Bezug auf eine Coronaviruskrankheit-2019 (COVID-19) sind nach Infektionsschutzgesetz (IfSG) meldepflichtig [3]. Die folgende Analyse basiert auf allen Fällen, die der Referenzdefinition (Nachweis von SARS-CoV-2 mittels Polymerasekettenreaktion (PCR) oder Kultur, unabhängig von der Art der klinischen Symptomatik [3]) des RKI entsprechen und die von den Gesundheitsämtern über die Landesgesundheitsbehörden an das RKI übermittelt wurden. Es wurden alle Fälle mit einem Meldedatum zwischen Meldewoche (MW) 5/2020 und 8/2021 sowie einer Angabe zum Alter berücksichtigt. Gemäß $\$ 11$ IfSG müssen auch Krankheitshäufungen von COVID-19 an das RKI übermittelt werden. Je nach regionaler Ausbreitung werden Ausbrüche entweder im Gesundheitsamt oder auf Bundesland- bzw. Bundesebene zu einem größeren Ausbruchsgeschehen zusammenge- 
Tab. 1 Einteilung der Schwere der Krankheitsverläufe basierend auf Angaben im Meldesystem gemäß IfSG [4] bzw. im ICOSARI-System [5]

\begin{tabular}{|c|c|c|}
\hline $\begin{array}{l}\text { Krankheits- } \\
\text { verlauf }\end{array}$ & Meldesystem gemäß IfSG & Krankenhaus-Sentinel ICOSARI \\
\hline Mild & $\begin{array}{l}\text { Angaben zum klinischen Bild vorhanden, } \\
\text { keine Pneumonie, nicht hospitalisiert, nicht } \\
\text { verstorben }\end{array}$ & Nicht verfügbar \\
\hline Moderat & $\begin{array}{l}\text { Angaben zum klinischen Bild vorhanden, } \\
\text { Pneumonie vorhanden, nicht hospitalisiert, } \\
\text { nicht verstorben }\end{array}$ & Nicht verfügbar \\
\hline Schwer & $\begin{array}{l}\text { Hospitalisiert (unabhängig von klinischen } \\
\text { Informationen, Intensivpflicht und Verster- } \\
\text { ben) }\end{array}$ & $\begin{array}{l}\text { Hospitalisiert (COVID-SARI-Fäl- } \\
\text { le, definiert über ICD-10-Codes } \\
\text { U07.1!+ J09-J22) }\end{array}$ \\
\hline \multirow[t]{2}{*}{ Kritisch } & \multirow[t]{2}{*}{ nicht angewendet } & $\begin{array}{l}\text { Intensivpflichtig (COVID-SARI-Fälle } \\
\text { mit Intensivbehandlung während der } \\
\text { Hospitalisierung) }\end{array}$ \\
\hline & & $\begin{array}{l}\text { Beatmet (COVID-SARI-Fälle mit me- } \\
\text { chanischer Beatmung während der } \\
\text { Hospitalisierung) }\end{array}$ \\
\hline Verstorben & $\begin{array}{l}\text { Verstorben (unabhängig von klinischen } \\
\text { Informationen und Hospitalisierung) }\end{array}$ & $\begin{array}{l}\text { Verstorben (COVID-SARI-Fälle, die } \\
\text { während ihrer Hospitalisierung ver- } \\
\text { storben sind) }\end{array}$ \\
\hline
\end{tabular}

fasst. In der folgenden Auswertung wurde die Anzahl der Ausbrüche (mit mindestens 2 Fällen) auf der jeweils höchsten Ausbruchsebene betrachtet [7]. Als wahrscheinliche Infektionsumfelder der Ausbrüche wurden die häufigsten Ausbruchssettings angegeben [8].

\section{Meldungen aus dem Krankenhaus- Sentinel (ICOSARI)}

Im Rahmen der seit 2015 bestehenden wissenschaftlichen Kooperation mit der HELIOS Kliniken GmbH hat das RKI ein kontinuierliches syndromisches Sentinel-Krankenhaus-Surveillance-System für schwere akute respiratorische Infektionskrankheiten (SARI) aufgebaut [5]. Das Sentinel umfasst dabei etwa $6 \%$ aller Krankenhausfälle in Deutschland [9]. Im Rahmen dieses Systems werden anonymisiert Daten von stationär aufgenommenen Patienten erhoben (ICOSARI: ICD-10-code-basierte KrankenhausSurveillance schwerer akuter respiratorischer Infektionen). Dabei wurden COVID-SARI-Fälle definiert als Fälle mit einem Labornachweis für SARSCoV-2 (ICD-10-Code U07.1!: COVID19, Virus nachgewiesen) sowie einer SARI (ICD-10-Codes J09-J22: Influenza sowie akute respiratorische Erkrankun- gen der unteren Atemwege). Für die deskriptive Analyse wurden COVIDSARI-Fälle betrachtet, die im Zeitraum von der Kalenderwoche (KW) 10/2020 bis zur KW 8/2021 in 72 Krankenhäusern des ICOSARI-Sentinels aufgenommen und von denen bis zum Datenstand 11.05.2021 eine Krankenhausentlassung, Verlegung oder ein Versterben übermittelt wurde. 7 Fälle, die zum Datenstand noch hospitalisiert waren, wurden von den Analysen ausgeschlossen.

\section{Deskriptive Analysen}

Die folgende Auswertung fokussiert sich auf die geografische und demografische Verteilung sowie die Krankheitsschwere im zeitlichen Verlauf. Die Entwicklung wurde anhand der gemeldeten COVID19-Fallzahlen gemäß IfSG nach Meldewoche (MW) dargestellt. Dies entspricht der Kalenderwoche, in der ein gemeldeter Fall beim Gesundheitsamt erfasst wurde. Die COVID-SARI-Fälle im ICOSARISentinel wurden entsprechend der Kalenderwoche (KW) erfasst, in der sie stationär aufgenommen wurden.

Für die Beschreibung der verschiedenen Krankheitsverläufe wurden milde, moderate, schwere und kritische Verläufe und Todesfälle unterschieden (•Tab. 1), wobei sich schwere und kritische Verläufe sowie Todesfälle nicht gegenseitig ausschließen. Diese Differenzierung basiert auf der initialen Verlaufsbeschreibung durch die Weltgesundheitsorganisation (WHO; [10]) und einer Bewertung der Krankheitsschwere basierend auf den Meldedaten gemäß IfSG $[4,11]$.

Für die Auswertung der gemäß RKIReferenzdefinition übermittelten Fälle in Bezug auf Krankheitsverlauf (mild, moderat) und Symptomatik wurden nur Fälle berücksichtigt, bei denen grundsätzlich eine Angabe zum klinischen Bild (Ja, Nein) vorlag.

Die Analyse wurde mithilfe von Microsoft Excel Professional 2019, QGIS Geographic Information System 3.14.1Pi sowie StataSE Version 15 durchgeführt. Für die Berechnung von Inzidenzen wurde die Bevölkerungsstatistik mit Stand 31.12.2019 herangezogen [12].

\section{Ergebnisse}

\section{Zeitliche und geografische Ausbreitung}

Zwischen MW 5/2020 und MW 8/2021 wurden insgesamt 2.444.983 COVID-19Fälle übermittelt (•Tab. 2). Im Zeitraum von MW 5/2020 bis 9/2020 (Phase 0) beschränkte sich das Infektionsgeschehen zunächst auf einzelne Stadt- und Landkreise (•Abb. 1). Hierbei war die mittlere wöchentliche Inzidenz mit 5,8 pro 100.000 im Landkreis Heinsberg (Nordrhein-Westfalen) am höchsten.

Ab MW 10/2020 folgte dann die erste COVID-19-Welle in Deutschland (Phase 1, • Abb. 2). Ab MW 11/2020 wurden erstmals aus allen Bundesländern Fälle berichtet. Zur gleichen Zeit wurden die infektionshygienischen Maßnahmen zur Kontaktbeschränkung verschärft und $\mathrm{zu}$ einem umfassenden Lockdown in KW 13/2020 ausgeweitet. Insbesondere die südlichen Landkreise in Bayern und Baden-Württemberg waren von hohen Fallzahlen betroffen (• Abb. 1). Bundesweit wurde der Höchstwert der wöchentlichen Inzidenz in MW 14/2020 mit 43 pro 100.000 erreicht (- Tab. 2). Mit dem Ende der ersten COVID-19Welle in MW 20/2020 wurden die kon- 
taktbeschränkenden Maßnahmen weiter sukzessive gelockert (- Abb. 2).

Im Zeitraum von MW 21/2020 bis 39/2020 (Phase 2) nahmen die Fallzahlen zunächst weiterhin kontinuierlich ab und sanken in MW 24/2020 auf die niedrigste wöchentliche Inzidenz von 2,8 pro 100.000 (• Abb. 2; • Tab. 2). Ab Mitte der Phase 2 kam es zu einem kontinuierlichen Anstieg der Fallzahlen. Auffällig waren hierbei die Landkreise Gütersloh (mittlere wöchentliche Inzidenz 35 pro 100.000) und Dingolfing-Landau (mittlere wöchentliche Inzidenz 42 pro 100.000; - Abb. 1). Parallel dazu wurden ab MW 31/2020 kostenlose Tests für aus dem Ausland Einreisende ermöglicht (• Abb. 2).

Mit Beginn des Herbstes kam es ab MW 40/2020 zu einem sprunghaften Anstieg der wöchentlichen Fallzahlen (Phase 3, -Abb. 2). Kurze Zeit später - in MW 42/2020 - folgte eine Anpassung der Teststrategie und damit eine Ausweitung der Testungen auf AntigenPoint-of-Care-Tests in Alten- und Pflegeheimen und Krankenhäusern. Dem weiteren Anstieg der Fallzahlen folgten in MW 45/2020 ein Teillockdown mit weiteren Kontaktbeschränkungen und ab Dezember 2020 weitere Verschärfungen und Anpassungen der Teststrategie. Nach dem Höhepunkt der zweiten Welle in MW 51/2020 mit einer wöchentlichen Inzidenz von 210 pro 100.000 sanken die Fallzahlen ab MW 1/2021 kontinuierlich bis MW 6/2021 und stagnierten bis MW $8 / 2021$ auf hohem Niveau (•Tab. 2). Wenige Kreise $(5 ; 1,2 \%)$ überschritten eine mittlere wöchentliche Inzidenz von 250 pro 100.000 (• Abb. 2). Dies waren insbesondere Kreise im Raum Sachsen an der Grenze zur Tschechischen Republik. Bundesweit lag die mittlere wöchentliche Inzidenz in dieser Phase bei 118 Fällen pro 100.000 (• Tab. 2).

\section{Expositionsorte und Ausbrüche}

Der Anteil der Fälle mit einer wahrscheinlichen Exposition im Ausland war vor allem in Phase $0(16 \%)$ und Phase 2 (23\%) erhöht und nahm während der COVID-19-Wellen in Phase 1 $(9,3 \%)$ und Phase 3 (1,0\%) deutlich ab (• Tab. 2).

Bundesgesundheitsbl 2021 · 64:1093-1106 https://doi.org/10.1007/s00103-021-03394-x (c) Der/die Autor(en) 2021

J. Schilling · K. Tolksdorf · A. Marquis - M. Faber · T. Pfoch · S. Buda - W. Haas · E. Schuler . D. Altmann · U. Grote · M. Diercke · RKI COVID-19 Study Group

\section{Die verschiedenen Phasen der COVID-19-Pandemie in Deutschland: Eine deskriptive Analyse von Januar 2020 bis Februar 2021}

\section{Zusammenfassung}

Am 27.01.2020 wurde in Deutschland der erste Fall mit einer SARS-CoV-2-Infektion diagnostiziert. Für die Beschreibung des Pandemieverlaufs im Jahr 2020 wurden 4 epidemiologisch verschiedene Phasen betrachtet und Daten aus dem Meldesystem gemäß Infektionsschutzgesetz (IfSG) sowie hospitalisierte COVID-19-Fälle mit schwerer akuter respiratorischer Infektion aus der Krankenhaus-Surveillance eingeschlossen. Phase 0 umfasst den Zeitraum von Kalenderwoche (KW) $5 / 2020$ bis $9 / 2020$, in dem vor allem sporadische Fälle $<60$ Jahre und regional begrenzte Ausbrüche beobachtet wurden. Insgesamt wurden 167 Fälle übermittelt, die vorwiegend mild verliefen. Dem schloss sich in Phase 1 (KW 10/2020 bis 20/2020) die erste COVID-19-Welle mit 175.013 Fällen im gesamten Bundesgebiet an. Hier wurden vermehrt Ausbrüche in Krankenhäusern, Alten- und Pflegeheimen sowie ein zunehmender Anteil an älteren und schwer erkrankten Personen verzeichnet.
In Phase 2, dem "Sommerplateau“ mit eher milden Verläufen (KW 21/2020 bis 39/2020), wurden viele reiseassoziierte COVID-19-Fälle im Alter von 15-59 Jahren und einzelne größere, überregionale Ausbrüche in Betrieben beobachtet. Unter den 111.790 Fällen wurden schwere Verläufe seltener beobachtet als in Phase 1. Phase 3 (KW 40/2020 bis 8/2021) war gekennzeichnet durch die zweite COVID-19-Welle in Deutschland, die sich zum Jahresende 2020 auf dem Höhepunkt befand. Mit 2.158.013 übermittelten COVID-19-Fällen und insgesamt deutlich mehr schweren Fällen in allen Altersgruppen verlief die zweite Welle schwerer als die erste Welle. Unabhängig von den 4 Phasen waren v. a. Ältere und auch Männer stärker von einem schweren Krankheitsverlauf betroffen.

Schlüsselwörter

Pandemie - SARS-CoV-2 - Epidemiologie . Meldesystem · Syndromische Surveillance

\section{The different periods of COVID-19 in Germany: a descriptive analysis from January 2020 to February 2021}

\section{Abstract}

The first case of coronavirus SARS-CoV-2 infection in Germany was diagnosed on $27 \mathrm{Ja}$ nuary 2020. To describe the pandemic course in 2020, we regarded four epidemiologically different periods and used data on COVID-19 cases from the mandatory reporting system as well as hospitalized COVID-19 cases with severe acute respiratory infection from the syndromic hospital surveillance.

Period 0 covers weeks 5 to 9 of 2020, where mainly sporadic cases of younger age were observed and few regional outbreaks emerged. In total, 167 cases with mostly mild outcomes were reported. Subsequently, the first COVID-19 wave occurred in period 1 (weeks 10 to 20 of 2020) with a total of 175,013 cases throughout Germany. Increasingly, outbreaks in hospitals and nursing homes were registered. Moreover, elderly cases and severe outcomes were observed more frequently. Period 2 (weeks 21 to 39 of
2020) was an interim period with more mild cases, where many cases were younger and often travel-associated. Additionally, larger trans-regional outbreaks in business settings were reported. Among the 111,790 cases, severe outcomes were less frequent than in period 1. In period 3 (week 40 of 2020 to week 8 of 2021), the second COVID-19 wave started and peaked at the end of 2020. With $2,158,013$ reported cases and considerably more severe outcomes in all age groups, the second wave was substantially stronger than the first wave.

Irrespective of the different periods, more elderly persons and more men were affected by severe outcomes.

\section{Keywords}

Pandemics - SARS-CoV-2 - Epidemiology . Mandatory surveillance $\cdot$ Syndromic surveillance 
Tab. 2 Angaben zu Alter, Geschlecht, Expositionsort und Ausbrüchen mit mind. 2 Fällen nach Phasen im Zeitraum Meldewoche (MW) 5/2020 bis 8/2021. (Quelle: an das RKI übermittelte COVID-19-Fälle (Datenstand: 11.05.2021), eigene Darstellung)

\begin{tabular}{|c|c|c|c|c|c|}
\hline & Gesamt & $\begin{array}{l}\text { Phase 0, } \\
\text { sporadische Fälle }\end{array}$ & $\begin{array}{l}\text { Phase } 1 \\
\text { Erste Welle }\end{array}$ & $\begin{array}{l}\text { Phase } 2 \\
\text { Sommerplateau }\end{array}$ & $\begin{array}{l}\text { Phase } 3 \\
\text { Zweite Welle }\end{array}$ \\
\hline Gesamtzahl Fälle & 2.444 .983 & 167 & 175.013 & 111.790 & 2.158 .013 \\
\hline Inzidenz (pro 100.000) & 2940 & 0,2 & 210 & 134 & 2595 \\
\hline $\begin{array}{l}\text { Mittlere wöchentl. Inz. } \\
\text { (pro 100.000) }\end{array}$ & 52 & 0,04 & 19 & 7,1 & 118 \\
\hline $\begin{array}{l}\text { Höchste wöchentl. Inz. } \\
\text { (pro 100.000) }\end{array}$ & 210 (MW 53/2020) & 0,2 (MW 9/2020) & $43(\mathrm{MW} 14 / 2020)$ & 16 (MW 39/2020) & 210 (MW 51/2020) \\
\hline $\begin{array}{l}\text { Niedrigste wöchentl. Inz. } \\
\text { (pro 100.000) }\end{array}$ & 0,01 (MW 5-8/2020) & 0,01 (MW 5-8/2020) & 1,08 (MW 10/2020) & 2,8 (MW 24/2020) & 19 (MW 40/2020) \\
\hline \multicolumn{6}{|c|}{ Altersgruppen (\%, Inzidenz pro 100.000) } \\
\hline Altersmedian (Jahre) & 44 & 36 & 50 & 33 & 45 \\
\hline 0-4 Jahre & $44.981(1,8 \%, 1135)$ & $6(3,6 \%, 0,2)$ & $1684(1,0 \%, 43)$ & $3477(3,1 \%, 88)$ & $39.814(1,8 \%, 1005)$ \\
\hline 5-14 Jahre & $136.938(5,6 \%, 1843)$ & $3(1,8 \%, 0,0)$ & $3956(2,3 \%, 53)$ & $9843(8,8 \%, 132)$ & $123.136(5,7 \%, 1657)$ \\
\hline 15-34 Jahre & $706.685(29 \%, 3696)$ & $71(43 \%, 0,4)$ & $43.719(25 \%, 229)$ & $46.168(41 \%, 241)$ & $616.727(29 \%, 3226)$ \\
\hline 35-59 Jahre & $930.393(38 \%, 3217)$ & $67(40 \%, 0,2)$ & $72.908(42 \%, 252)$ & $39.328(35 \%, 136)$ & $818.090(38 \%, 2829)$ \\
\hline 60-79 Jahre & $378.120(15 \%, 2094)$ & $19(11 \%, 0,1)$ & $32.968(19 \%, 183)$ & $9517(8,5 \%, 53)$ & $335.616(16 \%, 1859)$ \\
\hline$\geq 80$ Jahre & $247.866(10 \%, 4363)$ & $1(0,6 \%, 0,0)$ & $19.778(11 \%, 348)$ & $3457(3,1 \%, 61)$ & $224.630(10 \%, 3954)$ \\
\hline \multicolumn{6}{|c|}{ Geschlecht (\%, Inzidenz pro 100.000) } \\
\hline Weiblich & $1.283 .441(52 \%, 3046)$ & $80(48 \%, 0,2)$ & $91.449(52 \%, 217)$ & $52.568(47 \%, 125)$ & $1.139 .344(53 \%, 2704)$ \\
\hline Männlich & $1.145 .985(47 \%, 2793)$ & $87(52 \%, 0,2)$ & $83.416(48 \%, 203)$ & $58.793(53 \%, 143)$ & $1.003 .689(47 \%, 2446)$ \\
\hline Unbekannt & $15.557(0,6 \%)$ & - & $148(0,1 \%)$ & $429(0,4 \%)$ & $14.980(0,7 \%)$ \\
\hline \multicolumn{6}{|l|}{ Expositionsort } \\
\hline Deutschland & $1.413 .412(58 \%)$ & $117(70 \%)$ & $101.328(58 \%)$ & $56.759(51 \%)$ & $1.255 .208(58 \%)$ \\
\hline Ausland & $63.682(2,6 \%)$ & $26(16 \%)$ & $16.281(9,3 \%)$ & $25.880(23 \%)$ & $21.495(1,0 \%)$ \\
\hline Unbekannt & $967.889(40 \%)$ & $24(14 \%)$ & $57.404(33 \%)$ & $29.151(26 \%)$ & $881.310(41 \%)$ \\
\hline \multicolumn{6}{|l|}{ Ausbrüche } \\
\hline Ausbruchsfälle (\%) & $499.722(20 \%)$ & $116(70 \%)$ & $43.338(25 \%)$ & $37.328(33 \%)$ & $418.940(19 \%)$ \\
\hline $\begin{array}{l}\text { Altersmedian (Aus- } \\
\text { bruchsfälle) }\end{array}$ & 50 & 37 & 53 & 32 & 52 \\
\hline $\begin{array}{l}\text { Mittlere wöchentl. Anzahl } \\
\text { Ausbrüche }\end{array}$ & 1420 & 3,6 & 571 & 382 & 3063 \\
\hline $\begin{array}{l}\text { Häufigste Ausbruchsset- } \\
\text { tings }\end{array}$ & $\begin{array}{l}\text { Privater Haushalt, Alten- } \\
\text { und Pflegeheime, Arbeits- } \\
\text { platz }\end{array}$ & $\begin{array}{l}\text { Privater Haushalt, } \\
\text { Arbeitsplatz, Hotel/ } \\
\text { Herberge/Pension }\end{array}$ & $\begin{array}{l}\text { Privater Haushalt, Al- } \\
\text { ten- und Pflegeheime, } \\
\text { Krankenhäuser, Ar- } \\
\text { beitsplatz }\end{array}$ & $\begin{array}{l}\text { Privater Haushalt, } \\
\text { Arbeitsplatz, Frei- } \\
\text { zeit }\end{array}$ & $\begin{array}{l}\text { Privater Haushalt, Alten- } \\
\text { und Pflegeheime, Arbeits- } \\
\text { platz }\end{array}$ \\
\hline
\end{tabular}

Über alle Phasen hinweg wurden vor allem Ausbrüche im privaten Haushalt und am Arbeitsplatz berichtet. Wurden zu Beginn der Pandemie in Deutschland (Phase 0) neben diesen noch Übernachtungsmöglichkeiten (z.B. Hotel, Herberge, Pension) als häufigstes Ausbruchssetting angegeben, verschob sich der Schwerpunkt in der ersten COVID19-Welle zunehmend in Alten- und Pflegeheime sowie Krankenhäuser. Mit Beginn der Urlaubs- und Ferienzeit in Phase 2 wurden auch Freizeitaktivitäten als häufigste Ausbruchssettings genannt. In Phase 3 waren neben dem privaten
Haushalt erneut Alten- und Pflegeheime und der Arbeitsplatz häufige Ausbruchssettings. Der Altersmedian von Fällen in Ausbrüchen lag in den Phasen 0 und 2 deutlich niedriger (37 bzw. 32 Jahre) als während der beiden COVID-19-Wellen in den Phasen 1 und 3 (53 bzw. 52 Jahre).

\section{Krankheitsschwere}

\section{Demografie}

Der Anteil weiblicher und männlicher Fälle war gleich verteilt und variierte im Verlauf des Jahres nur um wenige Prozentpunkte (• Tab. 2). Insgesamt wurden vor allem COVID-19-Fälle im Alter von 35 bis 59 Jahren übermittelt (38\%). Diese Altersgruppe machte in jeder Phase den größten Anteil unter den übermittelten Fällen aus. Mit einer altersspezifischen Inzidenz von 4363 pro $100.000 \mathrm{im}$ gesamten Beobachtungszeitraum wurde jedoch die Altersgruppe der Personen ab 80 Jahren am häufigsten diagnostiziert. Mit Blick auf die Phasen war insbesondere während der COVID-19-Wellen (Phase 1 und 3) in dieser Altersgruppe die höchste Inzidenz zu beobachten. In den Interimsphasen (Phase 0 und 2) wurde dagegen die Altersgruppe der 15- bis 


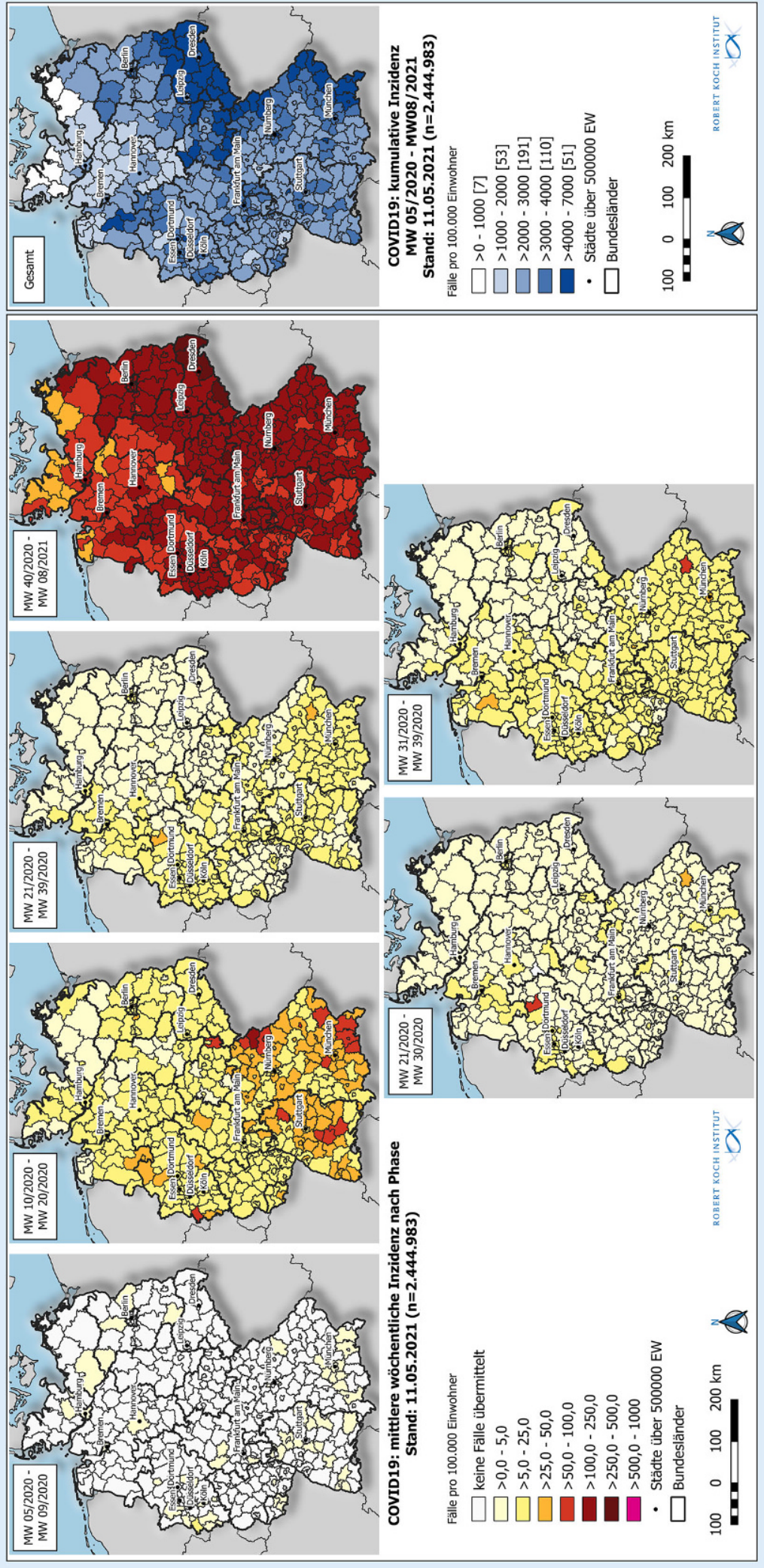

Abb. 1 ॥ Geografische Verteilung der übermittelten Fälle gemäß IfSG. Links: Mittlere wöchentliche Inzidenz nach Phase. Rechts: Kumulative Inzidenz für den Auswertungszeitraum Meldewochen 5/2020 bis 8/2021 in Deutschland. (Quelle: an das RKI übermittelte COVID-19-Fälle gemäßIfSG (Datenstand 11.05.2021), eigene Darstellung). IfSG Infektionsschutzgesetz
34-Jährigen häufiger diagnostiziert. Der höchste Altersmedian lag mit 50 Jahren in Phase 1, der niedrigste mit 33 Jahren in Phase 2.

\section{Klinik}

Die 3 häufigsten übermittelten Symptome waren Allgemeinbeschwerden (z.B. Schwäche, allgemeines Krankheitsgefühl, Gliederschmerzen; 49\%), Husten (41\%) und Fieber (29\%). In der Altersgruppe 0 bis 4 Jahre wurde am häufigsten Fieber (35\%) angegeben, wohingegen in den übrigen Altersgruppen Allgemeinbeschwerden am häufigsten genannt wurden (•Tab. 3). Unter allen Fällen mit übermittelten Symptomen wurden im Median 2 Symptome je Fall angegeben, jedoch variierte die Anzahl der genannten Symptome zwischen den Altersgruppen. So wurden für Kinder und Hochaltrige eher keine Symptome angegeben. Unter Fällen mit nur einem Symptom wurden vor allem Allgemeinbeschwerden genannt. Darüber hinaus traten bei Kindern (0-4 und 5-14 Jahre) vor allem Fieber und Schnupfen als einziges Symptom auf. Unter den Erwachsenen (15-59 Jahre) wurden dagegen eher Husten und Schnupfen bzw. bei Senioren ( $a b 60$ Jahre) eher Husten und Fieber genannt.

\section{Krankheitsverläufe}

Die Krankheitsverläufe werden im Folgenden basierend auf den übermittelten Fällen gemäß IfSG sowie der Krankenhaus-Surveillance ICOSARI beschrieben.

Insgesamt waren laut Meldesystem gemäß IfSG 1.337.428 Fälle (77\%) mild erkrankt, 192.191 Fälle (10\%) stationär aufgenommen worden und 75.402 Fälle (3,1\%) verstorben (• Tab. 4). Mit zunehmendem Alter stieg der Anteil an hospitalisierten und verstorbenen Fällen. So war die Mehrheit der Fälle unter 60 Jahren nur mild erkrankt. Dagegen hatte weniger als die Hälfte der Fälle ab 80 Jahren einen milden Verlauf und es wurde in dieser Altersgruppe der höchste Anteil hospitalisierter bzw. verstorbener Fälle beobachtet. Entsprechend lag der Altersmedian bei milden Fällen deutlich niedriger als bei hospitalisierten und verstorbenen Fällen (41 Jahre vs. 73 bzw. 84 Jahre). Im 


\section{Leitthema}

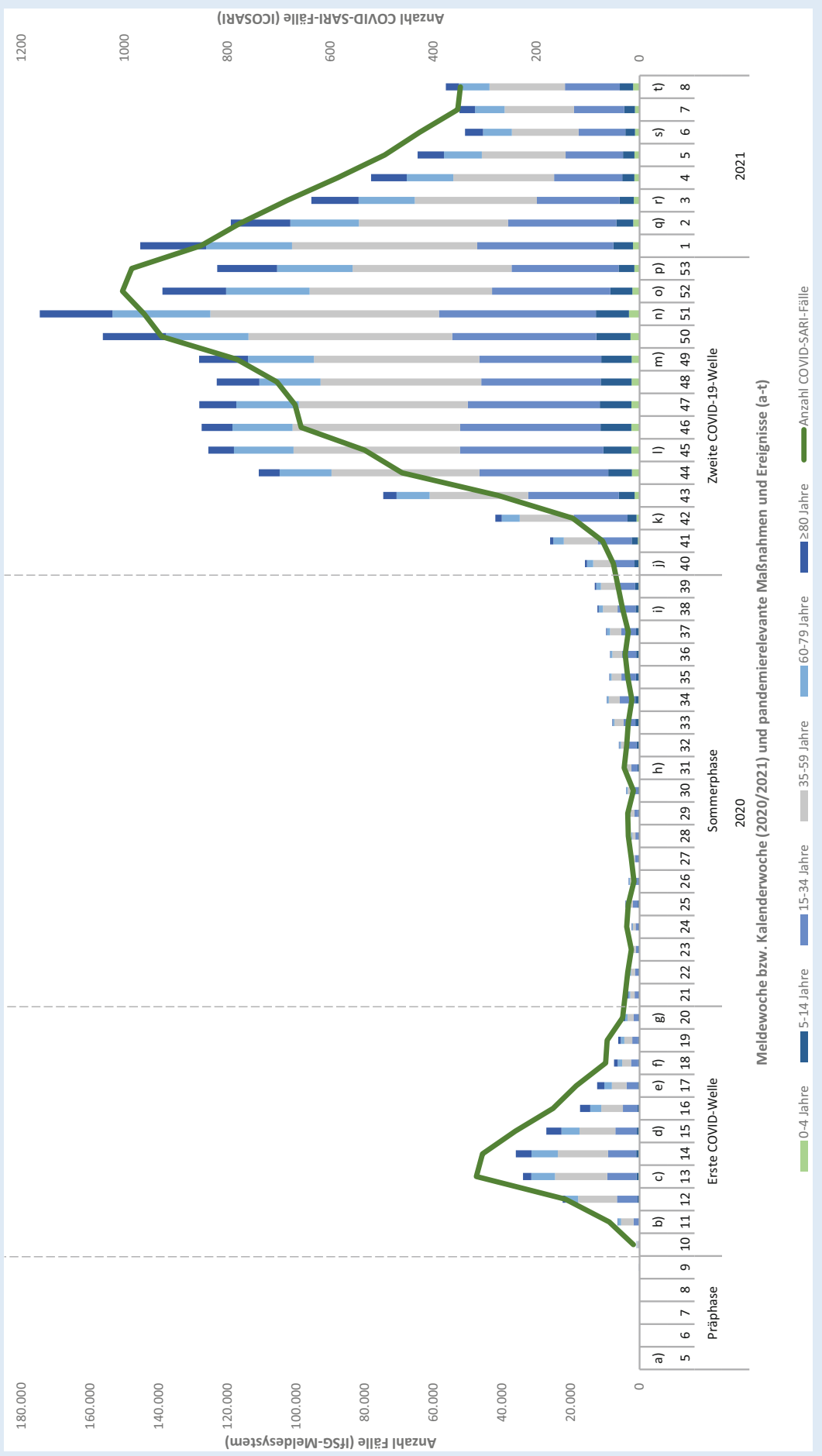


Abb. $2 \triangleleft$ Anzahl der übermittelten COVID-19-Fälle in Deutschland je Altersgruppe nach Meldewoche ( $n=2.444 .983)$ sowie wöchentliche Gesamtzahl der COVID-SARI-Fälle nach Kalenderwoche $(K W ; n=14.703)$ unter Angabe pandemierelevanter Maßnahmen und Ereignisse von KW 5/2020 bis $8 / 2021^{\mathrm{a}-\mathrm{t}}$. (Quelle: an das RKI übermittelte COVID-19-Fälle (Datenstand 11.05.2021) und COVIDSARI-Fälle aus der syndromischen Krankenhaus-Surveillance ICOSARI (Datenstand: 11.05.2021), eigene Darstellung). Hinweis: Die gemäß IfSG übermittelten Fälle werden nach Meldewoche dargestellt und die Fälle aus der syndromischen Krankenhaus-Surveillance nach Kalenderwoche. ${ }^{2} \mathrm{KW}$ 05/2020: 30.01.2020 - Weltgesundheitsorganisation (WHO) erklärt den COVID-19-Ausbruch zu einer gesundheitlichen Notlage internationaler Tragweite (PHEIC; [40]). ${ }^{\text {}} \mathrm{KW} 11 / 2020: 10.03 .2020$ Absage von Veranstaltungen mit mehr als 1000 Personen (beschlossen am 08.03., gültig ab 10.03.; [41]); 11.03.2020 - die WHO erklärt den COVID-19-Ausbruch zu einer Pandemie [40]; 15.03 .2020 es werden sukzessive Kitas und Schulen geschlossen [42]. KW 13/2020:23.03.2020 - bundesweiter Lockdown mit umfassenden Kontaktbeschränkungen und weitreichenden Schließungen, u. a. von Gastronomie [43]. ${ }^{\mathrm{K}} \mathrm{KW}$ 15/2020: 08.04.2020 - verpflichtende Quarantäne für Einreisende [44]. eKW 17/2020: 20.04.2020 - Beginn von ersten Lockerungsmaßnahmen (Einkaufen in Geschäften bis $800 \mathrm{~m}^{2}$, z. T. Wiederaufnahme des Schulbetriebs; [45, 46]). fKW 18/2020: 29.04.2020 - bundesweite Pflicht zum Tragen einer Mund-Nasen-Bedeckung (meist in Geschäften und im öffentlichen Personennahverkehr (ÖPNV); [47]); 30.04.2020 - weitere sukzessive Lockerung der Kontaktbeschränkungen (Öffnung von Spielplätzen, Museen, Zoos und Gotteshäusern; [48]). 9KW 20/2020: 11.05.2020 - sukzessive Öffnung der Gastronomie [49]. ' $K$ KW 31/2020: 01.08.2020 - kostenlose Tests für Einreisende (verpflichtende Testung für Einreisende aus Risikogebieten seit 08.08.2020; [50, 51]). . KW 38/2020: 15.09.2020 - Einstellung kostenloser Tests für Einreisende aus Nichtrisikogebieten [21]. .KW 40/2020: 01.10.2020 - es gelten wieder differenzierte Reise- und Sicherheitshinweise und Reisewarnungen für einzelne Länder. Eine Reisewarnung gilt für Länder mit einer 7-Tage-Inzidenz von 50 pro 100.000 [51, 52]. ${ }^{k}$ KW 42/2020: 15.10.2020 - Anpassung der Nationalen Teststrategie (Einführung von kostenlosen Antigenschnelltests in Alten- und Pflegeheimen sowie Krankenhäusern; [53]). 'KW 45/2020: 02.11.2020 - bundesweiter "Teillockdown“ mit verschärften Kontaktbeschränkungen [54]. mKW 49/2020: 01.12.2020 - Coronavirus-Testverordnung (Ausweitung des Testanspruchs in ausgewählten Settings bzw. für Kontaktpersonen auch auf asymptomatische Personen; z. T. auch als Point-ofCare-Test möglich) und teilweise Verschärfung der Maßnahmen vom 02.11.2020 [55]. "KW 51/2020: 16.12.2020 - Inkrafttreten des bundesweiten Lockdowns mit z. T. verschärften Regelungen [56]. ${ }^{\circ} \mathrm{KW}$ 52/2020: 21.12.2020 - Berichte zu neuen Virusmutationen (Variants of Concern, VOC) im Vereinigten Königreich und Südafrika; 27.12.2020 - Start der ersten Impfungen in Alten- und Pflegeheimen

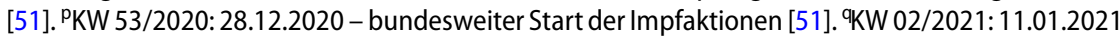
- Verlängerung und Verschärfung der Kontaktbeschränkungen [57]; 12.01.2021 - Hinweise auf eine neue Virusmutation in Brasilien [58]; 14.01.2021 - neue Einreise-Verordnung tritt in Kraft (Testpflicht innerhalb von $48 \mathrm{~h}$ nach Einreise; bei Einreise aus besonders betroffenen Regionen muss schon vor Einreise ein negatives Testergebnis vorliegen; [51]). 'KW 03/2021: 19.01.2021 - Verlängerung und Verschärfung der infektionshygienischen Maßnahmen (Verpflichtung zum Tragen sogenannter medizinischer Masken (in ÖPNV und Geschäften gilt eine FFP2/(K)N95-Maskenpflicht) sukzessive ab 20.01.2021, Homeofficepflicht sofern möglich [59]; Coronavirus-Surveillance-VO tritt in Kraft (5\% aller Positivtestungen sollen sequenziert werden; [60]). ${ }^{5}$ KW 06/2021: 10.02.2021 - Verlängerung der Maßnahmen bis 07.03.2021 (je nach lokaler Lage dürfen Schulen schrittweise öffnen; [61]); Anteil VOC B.1.1.7 liegt bei ca. $22 \%$ [39]. TKW 08/2021: VOC B.1.1.7 hat inzwischen einen Anteil von ca. $50 \%$ unter den gemeldeten Fällen [39]

Vergleich zwischen der ersten und zweiten Welle (Phase 1 und 3) gab es hier nur wenige Unterschiede. In Phase 2 (Sommerplateau) war der Altersmedian hospitalisierter Fälle jedoch mit 58 Jahren deutlich niedriger.

In allen Altersgruppen wurde in Phase 1 der höchste Anteil an hospitalisierten und verstorbenen Fällen beobachtet. Die Mehrzahl der hospitalisierten Fälle in Phase 1 war im Alter von 60 bis 79 Jahren. Im Vergleich dazu war die Mehrzahl der Fälle in der zweiten Welle (Phase 3) 80 Jahre oder älter. Unter verstorbenen Fällen wurden
SARI-Fälle in eine andere medizinische Einrichtung verlegt.

Mit 54\% war die Mehrheit der Fälle männlich - insbesondere unter den intensivpflichtigen (62\%), beatmeten $(65 \%)$ und stationär verstorbenen Fällen (58\%). Das Geschlechterverhältnis je nach Krankheitsverlauf veränderte sich im Jahresverlauf nur um wenige Prozentpunkte.

Insgesamt wurden 4781 (33\%) Personen intensivmedizinisch behandelt und im Median 15 Tage hospitalisiert (- Tab.5). In Phase 2 wurden sie jedoch etwas länger stationär behandelt und waren im Median etwas jünger als in den Phasen 1 und 3. Darüber hinaus war in Phase 2 der Anteil der Intensivbehandlungen bei COVID-SARI-Fällen am niedrigsten. Beim Vergleich der Wellen lag der Anteil der intensivpflichtigen Fälle während der zweiten Welle (Phase 3) niedriger als während der ersten Welle (Phase 1), insbesondere in den Altersgruppen ab 60 Jahre.

Während ihrer stationären Behandlung wurden 2941 (20\%) COVID-SARIFälle mechanisch beatmet (• Tab. 5). Im Median waren diese Fälle 73 Jahre alt, hier gab es kaum Unterschiede zwischen den einzelnen Phasen. Beatmete Fälle waren insbesondere in den Phasen 1 und 2 im Median deutlich länger hospitalisiert als intensivpflichtige COVID-SARI-Fälle insgesamt. In Phase 3 waren mechanisch beatmete Patienten im Median dagegen kaum länger hospitalisiert als intensivpflichtige COVID-SARI-Fälle. Hierbei zeigte sich vor allem ein Unterschied bei beatmeten Patienten mit einer späteren Verlegung in ein anderes Krankenhaus: Wurden diese Patienten in der ersten Welle im Median nach 27 Tagen verlegt, so erfolgte die Verlegung in der zweiten Welle bereits im Median nach 18 Tagen. Beatmungspflichtige Patienten ohne Verlegung waren dagegen in beiden Wellen ähnlich lange hospitalisiert (17 bzw. 16 Tage).

Während ihres Krankenhausaufenthalts verstarben 3752 (26\%) der COVIDSARI-Fälle (•Tab. 5). Hierbei lag der Altersmedian bei 82 Jahren und damit deutlich über dem Median der COVIDSARI-Patienten insgesamt. Während der beiden Wellen verstarben auch mehrere 
Tab. 3 Verteilung der Symptome und Symptomanzahl nach Altersgruppen (Mehrfachnennungen möglich). (Quelle: an das RKIübermittelte COVID19-Fälle (Datenstand: 11.05.2021), eigene Darstellung)

\begin{tabular}{|c|c|c|c|c|c|c|c|c|c|c|c|c|c|c|}
\hline \multirow{3}{*}{$\begin{array}{l}\text { Altersgruppen } \\
\text { Anzahl Fälle (n) } \\
\text { Anzahl Nennungen }\end{array}$} & \multicolumn{2}{|l|}{ Gesamt } & \multicolumn{2}{|c|}{ 0-4 Jahre } & \multicolumn{2}{|c|}{ 5-14 Jahre } & \multicolumn{2}{|c|}{ 15-34 Jahre } & \multicolumn{2}{|c|}{ 35-59 Jahre } & \multicolumn{2}{|c|}{ 60-79 Jahre } & \multicolumn{2}{|c|}{$\geq 80$ Jahre } \\
\hline & \multicolumn{2}{|l|}{2.444 .983} & \multicolumn{2}{|l|}{44.981} & \multicolumn{2}{|l|}{136.938} & \multicolumn{2}{|l|}{706.885} & \multicolumn{2}{|l|}{930.393} & \multicolumn{2}{|l|}{378.120} & \multicolumn{2}{|l|}{247.866} \\
\hline & $n$ & $\%$ & $n$ & $\%$ & $n$ & $\%$ & $n$ & $\%$ & $n$ & $\%$ & $n$ & $\%$ & $n$ & $\%$ \\
\hline \multicolumn{15}{|c|}{ Klinische Informationen vorhanden } \\
\hline Ja & 1.733 .666 & 71 & 29.590 & 66 & 89.737 & 66 & 511.851 & 72 & 689.379 & 74 & 267.312 & 71 & 145.800 & 59 \\
\hline Nein & 226.653 & 9,3 & 6542 & 15 & 20.380 & 15 & 57.166 & 8,1 & 70.774 & 7,6 & 36.358 & 9,6 & 35.433 & 14 \\
\hline Unbekannt & 484.664 & 20 & 8849 & 20 & 26.821 & 20 & 137.668 & 20 & 170.243 & 18 & 74.450 & 20 & 66.633 & 27 \\
\hline \multicolumn{15}{|l|}{ Symptome } \\
\hline $\begin{array}{l}\text { Allgemeine Krankheitszei- } \\
\text { chen }\end{array}$ & 851.705 & 49 & 5944 & 20 & 27.701 & 31 & 256.840 & 50 & 377.356 & 55 & 130.493 & 49 & 53.371 & 37 \\
\hline Husten & 702.233 & 41 & 8417 & 29 & 20.162 & 23 & 207.718 & 41 & 309.973 & 45 & 115.416 & 43 & 40.547 & 28 \\
\hline Schnupfen & 502.699 & 29 & 7882 & 27 & 21.535 & 24 & 180.860 & 35 & 216.747 & 31 & 60.091 & 23 & 15.584 & 11 \\
\hline Fieber & 463.364 & 27 & 10.222 & 35 & 18.815 & 21 & 128.770 & 25 & 200.259 & 29 & 73.950 & 28 & 31.348 & 22 \\
\hline Halsschmerzen & 370.516 & 21 & 2032 & 6,9 & 14.208 & 16 & 139.823 & 27 & 160.797 & 23 & 42.677 & 16 & 10.979 & 7,5 \\
\hline Geschmacksverlust & 308.273 & 18 & 499 & 1,7 & 6132 & 6,8 & 132.323 & 26 & 137.579 & 20 & 27.323 & 10 & 4417 & 3,0 \\
\hline Geruchsverlust & 280.860 & 16 & 363 & 1,2 & 5277 & 5,9 & 121.269 & 24 & 127.206 & 19 & 23.618 & 8,8 & 3127 & 2,1 \\
\hline Dyspnoe & 83.993 & 4,8 & 305 & 1,0 & 555 & 0,6 & 17.433 & 3,4 & 31.853 & 4,6 & 19.153 & 7,2 & 14.694 & 10 \\
\hline Durchfall & 80.956 & 4,7 & 1508 & 5,1 & 2474 & 2,8 & 19.826 & 3,9 & 34.596 & 5,0 & 15.889 & 5,9 & 6663 & 4,6 \\
\hline Pneumonie & 26.486 & 1,5 & 53 & 0,9 & 91 & 0,1 & 1447 & 0,3 & 5353 & 0,8 & 8836 & 3,3 & 10.706 & 7,3 \\
\hline ARDS $^{\mathrm{a}}$ & 14.415 & 0,8 & 42 & 0,1 & 110 & 0,1 & 2258 & 0,4 & 4500 & 0,7 & 4329 & 1,6 & 3176 & 2,2 \\
\hline Tachykardie & 5410 & 0,3 & 14 & 0,1 & 40 & 0,04 & 1153 & 0,2 & 2221 & 0,3 & 1199 & 0,5 & 783 & 0,5 \\
\hline Tachypnoe & 5846 & 0,3 & 23 & 0,1 & 42 & 0,1 & 1388 & 0,3 & 2443 & 0,4 & 1188 & 0,4 & 762 & 0,5 \\
\hline \multicolumn{15}{|l|}{ Anzahl Symptome } \\
\hline 1 Symptom & 334.209 & 19 & 7969 & 27 & 21.653 & 24 & 84.825 & 17 & 125.333 & 18 & 57.423 & 22 & 37.006 & 25 \\
\hline 2 Symptome & 397.990 & 23 & 6458 & 22 & 18.821 & 21 & 115.682 & 23 & 164.360 & 24 & 64.286 & 24 & 28.383 & 20 \\
\hline 3 Symptome & 324.692 & 19 & 3320 & 11 & 10.220 & 11 & 103.827 & 20 & 141.612 & 21 & 48.535 & 18 & 17.178 & 12 \\
\hline Mind. 4 Symptome & 338.080 & 20 & 1493 & 5,1 & 6058 & 6,8 & 122.845 & 24 & 154.298 & 22 & 41.847 & 16 & 11.539 & 7,9 \\
\hline Unbekannt & 338.695 & 20 & 10.350 & 35 & 32.985 & 37 & 84.672 & 17 & 103.773 & 15 & 55.221 & 21 & 51.694 & 36 \\
\hline
\end{tabular}

COVID-SARI-Fälle aus der Altersgruppe 35 bis 59 Jahre (4\% bzw. $5 \%)$ im Krankenhaus. In der zweiten Welle wurde insgesamt ein höherer Anteil Todesfälle berichtet als in der ersten Welle. In Phase 2 gab es den niedrigsten Anteil an Todesfällen unter den COVID-SARIFällen, jedoch wurden sie in dieser Phase im Median etwas länger hospitalisiert.

\section{Diskussion}

Insgesamt war das COVID-19-Infektionsgeschehen in Deutschland durch unterschiedliche Phasen geprägt, die durch 2 unterschiedlich schwer verlaufende COVID-19-Wellen im Frühjahr und Herbst und einer Sommerplateauphase mit eher milden Verläufen charakterisiert waren.
Der erste laborbestätigte Fall mit einer SARS-CoV-2-Infektion wurde in Deutschland in MW 5/2020 übermittelt $[1,13,14]$. Innerhalb der folgenden Wochen wurden weitere Fälle mit einer Exposition im Ausland bekannt. Darunter befanden sich aus China repatriierte Personen [14, 15], aber auch Einreisende aus Urlaubsgebieten (u.a. Skigebiete in Italien und Österreich; [16, 17]). Sie spielten zum Teil eine tragende Rolle für das Infektionsgeschehen auf regionaler Ebene [16], was den vergleichsweise hohen Anteil importierter Fälle zu Beginn der Pandemie erklärt. Mitte Februar folgten Ausbrüche in Zusammenhang mit lokalen Feiern (z. B. Karneval), die $\mathrm{zu}$ erhöhten Fallzahlen in einzelnen Kreisen führten (u.a. LK Heinsberg, LK Tirschenreuth; $[15,16,18,19])$ und den Beginn der ersten COVID-19-Welle einleiteten.

In der Phase 1 waren alle Bundesländer betroffen, wobei lokale Feiern und die Nähe zu Grenzregionen mit einem erhöhten Infektionsgeschehen eine wichtige Rolle für regionale Ausbreitung spielten $[14,20]$. Ausbruchsgeschehen traten nun auch vermehrt in stationären Einrichtungen auf. Laut Buda et al. sind stationäre Ausbruchssettings mit einer höheren Anzahl an Fällen assoziiert [8], was sich auch im zunehmenden Anteil an Fällen höheren Alters in Phase 1 widerspiegelte.

Mit dem Ende der ersten Welle begann die Phase 2, in der alle Bundesländer nur sehr niedrige Inzidenzen meldeten. Insgesamt gab es in Phase 2 nur wenige Vorgaben für kontaktreduzierende Maßnahmen. Dagegen wurden jedoch 
Tab.4 Altersspezifische Verteilung nach Krankheitsverlaufund Phase. (Quelle: an das RKIübermittelte COVID-19-Fälle (Datenstand 11.05.2021), eigene Darstellung)

\begin{tabular}{|c|c|c|c|c|c|c|c|c|c|c|c|c|c|c|c|}
\hline \multirow[b]{2}{*}{ Verlauf } & \multicolumn{3}{|l|}{ Gesamt } & \multicolumn{2}{|c|}{ 0-4 Jahre } & \multicolumn{2}{|c|}{ 5-14 Jahre } & \multicolumn{2}{|c|}{ 15-34 Jahre } & \multicolumn{2}{|c|}{ 35-59 Jahre } & \multicolumn{2}{|c|}{ 60-79 Jahre } & \multicolumn{2}{|c|}{$\geq 80$ Jahre } \\
\hline & Altersmedian & $n$ & $\%$ & $n$ & $\%$ & $n$ & $\%$ & $n$ & $\%$ & $n$ & $\%$ & $n$ & $\%$ & $n$ & $\%$ \\
\hline \multicolumn{16}{|l|}{ Gesamt (2020) } \\
\hline Mild & 41 & 1.337 .428 & 77 & 25.061 & 85 & 77.604 & 87 & 434.572 & 85 & 559.744 & 81 & 175.276 & 66 & 65.171 & 45 \\
\hline Moderat & 49 & 3388 & 0,2 & 26 & 0,1 & 64 & 0,1 & 809 & 0,2 & 1532 & 0,2 & 704 & 0,3 & 253 & 0,2 \\
\hline Hospitalisiert & 73 & 192.191 & 10 & 1402 & 4,0 & 1190 & 1,1 & 13.006 & 2,4 & 39.299 & 5,6 & 68.360 & 23 & 68.934 & 36 \\
\hline Verstorben & 84 & 75.402 & 3,1 & 5 & 0 & 5 & 0 & 99 & 0 & 2503 & 0,3 & 20.566 & 5,5 & 52.224 & 21 \\
\hline \multicolumn{16}{|c|}{ Phase 0, sporadische Fälle } \\
\hline Mild & 33 & 97 & 65 & 2 & a & 3 & a & 48 & 74 & 33 & 55 & 10 & a & 1 & a \\
\hline Moderat & k. A. & 0 & 0 & 0 & a & 0 & a & 0 & 0 & 0 & 0 & 0 & a & 0 & a \\
\hline Hospitalisiert & 40,5 & 48 & 32 & 3 & a & 3 & a & 16 & 25 & 24 & 41 & 5 & a & 0 & a \\
\hline Verstorben & 65 & 1 & 0,6 & 0 & a & 0 & a & 0 & 0 & 0 & 0 & 1 & a & 0 & a \\
\hline \multicolumn{16}{|c|}{ Phase 1, erste Welle } \\
\hline Mild & 46 & 111.188 & 72 & 1105 & 80 & 2810 & 86 & 32.545 & 85 & 52.412 & 80 & 16.544 & 57 & 5772 & 36 \\
\hline Moderat & 52 & 438 & 0,3 & 1 & 0,1 & 3 & 0,1 & 81 & 0,2 & 224 & 0,3 & 98 & 0,3 & 31 & 0,2 \\
\hline Hospitalisiert & 71 & 27.729 & 18 & 164 & 11 & 112 & 3,3 & 1684 & 4,5 & 6491 & 10 & 10.691 & 36 & 8587 & 48 \\
\hline Verstorben & 82 & 8912 & 5,1 & 1 & 0,1 & 0 & 0,0 & 19 & 0,0 & 406 & 0,6 & 2891 & 8,8 & 5595 & 28 \\
\hline \multicolumn{16}{|c|}{ Phase 2, Sommerplateau } \\
\hline Mild & 31 & 70.973 & 82 & 2202 & 87 & 6205 & 89 & 31.381 & 87 & 25.138 & 82 & 4990 & 66 & 1057 & 42 \\
\hline Moderat & 42 & 169 & 0,2 & 0 & 0 & 8 & 0,1 & 54 & 0,1 & 70 & 0,2 & 34 & 0,4 & 3 & 0,1 \\
\hline Hospitalisiert & 58 & 7648 & 8,0 & 122 & 4,1 & 121 & 1,4 & 1141 & 2,9 & 2586 & 7,7 & 2212 & 27 & 1466 & 47 \\
\hline Verstorben & 81 & 864 & 0,8 & 0 & 0 & 0 & 0 & 5 & 0 & 74 & 0,2 & 295 & 3,2 & 490 & 17 \\
\hline \multicolumn{16}{|c|}{ Phase 3, zweite Welle } \\
\hline Mild & 41 & 1.155 .170 & 77 & 21.752 & 85 & 68.586 & 86 & 370.598 & 85 & 482.161 & 81 & 153.732 & 67 & 58.341 & 46 \\
\hline Moderat & 49 & 2781 & 0,2 & 25 & 0,1 & 53 & 0,1 & 674 & 0,2 & 1238 & 0,2 & 572 & 0,2 & 219 & 0,2 \\
\hline Hospitalisiert & 74 & 156.766 & 9,7 & 1113 & 3,6 & 957 & 1,0 & 10.165 & 2,2 & 30.198 & 5,0 & 55.452 & 22 & 58.881 & 35 \\
\hline Verstorben & 84 & 65.625 & 3,1 & 4 & 0 & 5 & 0 & 75 & 0 & 2023 & 0,2 & 17.379 & 5,2 & 46.139 & 21 \\
\hline
\end{tabular}

zusätzliche, niedrigschwellige und kostenlose Testmöglichkeiten für aus dem Ausland Einreisende ab KW 31/2020 geschaffen, welche erst mit dem Ende der Sommerferien in allen Bundesländern in KW 38/2020 für Einreisende aus Nichtrisikogebieten eingestellt wurden [21]. Die zunehmende Reisetätigkeit in den Sommermonaten und die zunehmend zur Verfügung stehenden Testmöglichkeiten spiegelten sich u.a. im Anstieg von Fällen mit einer wahrscheinlichen Exposition im Ausland und der Anzahl an Ausbrüchen im Freizeitbereich wider. Im Vergleich zu den anderen Phasen wurde in Phase 2 der jüngste Altersmedian (33 Jahre) unter den übermittelten COVID-19-Fällen verzeichnet. Dies ist wahrscheinlich darauf zurückzuführen, dass diese reiseassoziierten Fälle ver- gleichsweise jünger waren und durch die sensitive Teststrategie an den Flughäfen im Sommer (im Vergleich zur Phase 0) auch (ebenfalls vermehrt junge) asymptomatische Fälle erkannt wurden [22]. Auch die großen Ausbrüche in Betrieben mit überwiegend jungen Beschäftigten (u. a. in Fleisch verarbeitenden Betrieben, wie bspw. in Gütersloh, oder unter Erntehelfern, wie bspw. im Kreis Dingolfing-Landau) trugen hierzu bei $[14,23$, 24].

Die Phase 3 war insbesondere geprägt durch autochthone Fälle sowie eine sechsmal höhere Anzahl an Ausbrüchen im Wochenmittel als in der ersten Welle. Wurden zu Beginn der Phase eher Fälle unter 60 Jahren diagnostiziert, nahmen im weiteren Verlauf mit der Anzahl an Ausbrüchen in statio- nären Einrichtungen auch die Fälle unter älteren Personen und damit auch unter Personen mit hohem Risiko für einen schweren Verlauf zu. Der hohe Anteil an unbekannten Expositionsorten und Ausbruchssettings spricht zudem für ein zunehmend diffuses Infektionsgeschehen, in dem Infektionsketten nur noch unzureichend ermittelt werden konnten.

Mit Blick auf die Krankheitsverläufe war der Anteil hospitalisierter Fälle mit $32 \%$ in Phase 0 am höchsten. Jedoch wurden in dieser Phase bestätigte COVID-19-Fälle zum Zweck der Beobachtung im Krankenhaus isoliert, daher spiegelt die hohe Hospitalisierungsquote hier eher eine infektionshygienische Maßnahme wider [11]. Dieses Vorgehen änderte sich zunehmend mit dem Anstieg der Fallzahlen und der steigenden Belas- 


\section{Leitthema}

Tab. 5 Altersspezifische Verteilung schwerer und kritischer Fälle nach Krankheitsverlauf und Phase. (Quelle: COVID-SARI-Fälle aus dem ICOSARI-Krankenhaus-Sentinel (Datenstand 11.05.2021))

\begin{tabular}{|c|c|c|c|c|c|c|c|c|c|c|c|c|}
\hline & \multicolumn{3}{|l|}{ Gesamt } & \multicolumn{3}{|c|}{ Phase 1} & \multicolumn{3}{|c|}{ Phase 2} & \multicolumn{3}{|c|}{ Phase 3} \\
\hline & $n$ & $\begin{array}{l}\% \text { an hos- } \\
\text { pitalisiert }\end{array}$ & $\begin{array}{l}\text { Medi- } \\
\text { an }\end{array}$ & $n$ & $\begin{array}{l}\% \text { an hos- } \\
\text { pitalisiert }\end{array}$ & $\begin{array}{l}\text { Medi- } \\
\text { an }\end{array}$ & $n$ & $\begin{array}{l}\% \text { an hos- } \\
\text { pitalisiert }\end{array}$ & $\begin{array}{l}\text { Medi- } \\
\text { an }\end{array}$ & $n$ & $\begin{array}{l}\% \text { an hos- } \\
\text { pitalisiert }\end{array}$ & $\begin{array}{l}\text { Medi- } \\
\text { an }\end{array}$ \\
\hline \multicolumn{13}{|l|}{ Hospitalisiert } \\
\hline Alter (Jahre) & - & - & 76 & - & - & 73 & - & - & 59 & - & - & 76 \\
\hline Dauer (Tage) $)^{b}$ & - & - & 9 & - & - & 10 & - & - & 9 & - & - & 9 \\
\hline 0-4 Jahre & 28 & $100 \%$ & - & 1 & a & - & 4 & a & - & 23 & $100 \%$ & - \\
\hline 5-14 Jahre & 9 & a & - & 1 & a & - & 2 & a & - & 6 & a & - \\
\hline 15-34 Jahre & 432 & $100 \%$ & - & 52 & $100 \%$ & - & 46 & $100 \%$ & - & 334 & $100 \%$ & - \\
\hline 35-59 Jahre & 2810 & $100 \%$ & - & 358 & $100 \%$ & - & 168 & $100 \%$ & - & 2284 & $100 \%$ & - \\
\hline 60-79 Jahre & 5694 & $100 \%$ & - & 615 & $100 \%$ & - & 137 & $100 \%$ & - & 4942 & $100 \%$ & - \\
\hline$\geq 80$ Jahre & 5730 & $100 \%$ & - & 504 & $100 \%$ & - & 81 & $100 \%$ & - & 5145 & $100 \%$ & - \\
\hline Gesamt & 14.703 & $100 \%$ & - & 1531 & $100 \%$ & - & 438 & $100 \%$ & - & 12.734 & $100 \%$ & - \\
\hline \multicolumn{13}{|c|}{ Intensivpflichtig } \\
\hline Alter (Jahre) & - & - & 73 & - & - & 72 & - & - & 70 & - & - & 74 \\
\hline Dauer (Tage) ${ }^{b}$ & - & - & 15 & - & - & 16 & - & - & 18,5 & - & - & 15 \\
\hline 0-4 Jahre & 2 & $7 \%$ & - & 0 & a & - & 2 & a & - & 0 & $0 \%$ & - \\
\hline 5-14 Jahre & 2 & a & - & 0 & a & - & 1 & a & - & 1 & a & - \\
\hline 15-34 Jahre & 66 & $15 \%$ & - & 8 & $15 \%$ & - & 3 & $7 \%$ & - & 55 & $16 \%$ & - \\
\hline 35-59 Jahre & 850 & $30 \%$ & - & 120 & $34 \%$ & - & 28 & $17 \%$ & - & 702 & $31 \%$ & - \\
\hline 60-79 Jahre & 2326 & $41 \%$ & - & 289 & $47 \%$ & - & 59 & $43 \%$ & - & 1978 & $40 \%$ & - \\
\hline$\geq 80$ Jahre & 1535 & $27 \%$ & - & 162 & $32 \%$ & - & 29 & $36 \%$ & - & 1344 & $26 \%$ & - \\
\hline Gesamt & 4781 & $33 \%$ & - & 579 & $38 \%$ & - & 122 & $28 \%$ & - & 4080 & $32 \%$ & - \\
\hline \multicolumn{13}{|c|}{ Mechanisch beatmet } \\
\hline Alter (Jahre) & - & - & 72 & - & - & 71 & - & - & 72 & - & - & 72 \\
\hline Dauer $(\text { Tage })^{\mathrm{b}}$ & - & - & 17 & - & - & 20 & - & - & 22 & - & - & 16 \\
\hline 0-4 Jahre & 2 & $7 \%$ & - & 0 & a & - & 2 & a & - & 0 & $0 \%$ & - \\
\hline 5-14 Jahre & 0 & a & - & 0 & a & - & 0 & a & - & 0 & a & - \\
\hline 15-34 Jahre & 31 & $7 \%$ & - & 5 & $10 \%$ & - & 0 & $0 \%$ & - & 26 & $8 \%$ & - \\
\hline 35-59 Jahre & 536 & $19 \%$ & - & 67 & $19 \%$ & - & 14 & $8 \%$ & - & 455 & $20 \%$ & - \\
\hline 60-79 Jahre & 1611 & $28 \%$ & - & 192 & $31 \%$ & - & 41 & $30 \%$ & - & 1378 & $28 \%$ & - \\
\hline$\geq 80$ Jahre & 761 & $13 \%$ & - & 74 & $15 \%$ & - & 18 & $22 \%$ & - & 669 & $13 \%$ & - \\
\hline Gesamt & 2941 & $20 \%$ & - & 338 & $22 \%$ & - & 75 & $17 \%$ & - & 2528 & $20 \%$ & - \\
\hline \multicolumn{13}{|l|}{ Verstorben } \\
\hline Alter (Jahre) & - & - & 82 & - & - & 81 & - & - & 80 & - & - & 82 \\
\hline Dauer $(\text { Tage })^{\mathrm{b}}$ & - & - & 10 & - & - & 10 & - & - & 13 & - & - & 10 \\
\hline 0-4 Jahre & 0 & $0 \%$ & - & 0 & a & - & 0 & a & - & 0 & $0 \%$ & - \\
\hline 5-14 Jahre & 0 & a & - & 0 & a & - & 0 & a & - & 0 & $a$ & - \\
\hline 15-34 Jahre & 4 & $1 \%$ & - & 2 & $4 \%$ & - & 0 & $0 \%$ & - & 2 & $1 \%$ & - \\
\hline 35-59 Jahre & 139 & $5 \%$ & - & 14 & $4 \%$ & - & 1 & $1 \%$ & - & 124 & $5 \%$ & - \\
\hline 60-79 Jahre & 1304 & $23 \%$ & - & 128 & $21 \%$ & - & 18 & $13 \%$ & - & 1158 & $23 \%$ & - \\
\hline$\geq 80$ Jahre & 2305 & $40 \%$ & - & 185 & $37 \%$ & - & 26 & $32 \%$ & - & 2094 & $41 \%$ & - \\
\hline Gesamt & 3752 & $26 \%$ & - & 329 & $21 \%$ & - & 45 & $10 \%$ & - & 3378 & $27 \%$ & - \\
\hline
\end{tabular}


tung der Krankenhauskapazitäten während der ersten Welle. Der im Vergleich zur zweiten Welle höhere Anteil schwerer und kritischer Fälle kann jedoch nur zum Teil auf die niedrigschwellige Hospitalisierung von COVID-19-Fällen zurückgeführt werden. Vielmehr ist von multiplen Einflüssen auszugehen.

Dabei ist beispielsweise die veränderte Teststrategie zu berücksichtigen. $\mathrm{Zu}$ Beginn der Phase 1 befanden sich die Testkapazitäten noch im Auf- bzw. Ausbau $[4,25,26]$. Mit der Einführung von Reihentestungen (z.B. am Flughafen ab KW 31/2020) und der Verfügbarkeit von Antigenschnelltests in Krankenhäusern und Alten- und Pflegeheimen ab KW 42/2020 (Phase 3) wurden in Deutschland jedoch auch Fälle mit nur geringer Symptomatik (mit und ohne schweren Verlauf) in den Meldedaten gemäß IfSG besser erfasst. Dagegen wurden in der syndromischen Krankenhaus-Surveillance ICOSARI in allen Phasen nur schwer erkrankte COVID-19-Fälle mit respiratorischer Symptomatik (SARI) betrachtet. Damit sind in diesem System stabilere Aussagen zum Anteil kritischer Verläufe in den verschiedenen Phasen möglich, unabhängig von der gewählten Teststrategie und den Empfehlungen zur niedrigschwelligen Krankenhausaufnahme.

Dennoch zeigte sich auch im ICOSARI-System ein etwas höherer Anteil intensivpflichtiger und beatmeter COVID-SARI-Fälle, was mit Erfahrungen aus europäischen Ländern und den USA übereinstimmt [27]. Dies wird insbesondere auf verbesserte Behandlungskonzepte u. a. durch eine verstärkte Nutzung nichtmechanischer Beatmung in späteren Phasen (insbesondere Phase 3) zurückgeführt [28-30]. Zudem wurde in Deutschland während der zweiten Welle ein System zur Verlegung von intensivmedizinisch behandelten $\mathrm{Pa}$ tienten etabliert, um die Überlastung einzelner Krankenhäuser zu vermeiden (Kleeblattsystem; [31]). Dies zeigte sich im ICOSARI-System in der kürzeren Hospitalisierungsdauer von beatmeten COVID-SARI-Patienten mit einer späteren Verlegung. Ergänzend zu diesen Punkten haben möglichweise auch die ab KW 53/2020 eingeführten Impfungen im Abklingen der zweiten Welle zu einer verringerten Krankheitsschwere in der Altersgruppe ab 80 Jahren beigetragen [32].

Die Altersverteilung bei kritischen Krankheitsverläufen blieb in den Phasen 1 bis 3 weitestgehend stabil. Während vor allem COVID-SARI-Fälle der Altersgruppe 60 bis 79 Jahre intensivmedizinisch behandelt oder beatmet wurden, war der Anteil verstorbener COVID-SARI-Fälle in der Altersgruppe ab 80 Jahre besonders hoch. Jedoch wurde deutlich, dass auch jüngere Altersgruppen von kritischen Verläufen betroffen waren: So wurde aus der Altersgruppe 35 bis 59 Jahre während der ersten und zweiten Welle jeder 3. hospitalisierte COVID-SARI-Patient intensivmedizinisch behandelt, jeder 5 . wurde mechanisch beatmet und etwa jeder 20 . COVID-SARI-Patient der Altersgruppe 35 bis 59 Jahre verstarb während des Krankenhausaufenthalts.

Insgesamt decken sich die Ergebnisse zur Altersverteilung mit den ersten Auswertungen zur Krankheitsschwere im Meldesystem bzw. unter hospitalisierten COVID-SARI-Fällen sowie mit Analysen im internationalen Vergleich [4, 11, 33-36]. So stellten auch Ioannidis et al. bei der Untersuchung von COVID-19-Todesfällen in Europa und den USA fest, dass sich die Altersverteilungen der ersten und zweiten COVID19-Welle kaum unterschieden und Alter ein wesentlicher Risikofaktor für einen schweren Verlauf ist [34].

Unabhängig von den Phasen wurden weibliche und männliche Fälle in ähnlichem Umfang übermittelt. Insgesamt waren jedoch männliche Fälle häufiger von schweren Verläufen betroffen, was sich insbesondere unter den intensivpflichtigen und den beatmungspflichtigen COVID-SARI-Fällen im Krankenhaus-Sentinel ICOSARI widerspiegelte. Dies wird zum Teil auf geschlechtsspezifische Alterungsprozesse im Immunsystem zurückgeführt, auch wenn hier noch weitere Analysen erforderlich sind [37].

\section{Limitationen}

Die vorliegende Auswertung basiert auf der Bewertung von Surveillance-Daten und unterliegt damit einigen Limitationen. Wie bereits vielfach beschrieben sind die Meldungen gemäß IfSG abhängig von den zugrunde liegenden Kapazitäten in Laboren, Gesundheitsämtern und Landesgesundheitsbehörden $[2,4]$ und damit anfällig für Verzögerungen. Durch den gewählten Datenstand (11.05.2021) wurde versucht, den Einfluss von Nachmeldungen - insbesondere zu schwer erkrankten Fällen zu reduzieren. Darüber hinaus hängen die Meldungen gemäß IfSG auch von der Inanspruchnahme der Testmöglichkeiten durch die Bevölkerung und der in Deutschland angewendeten Teststrategie ab. Dennoch deutet der bereits zu Beginn relativ hohe Anteil milder Fälle auf eine sensitive Teststrategie und eine gute Erfassung des Gesamtgeschehens im Meldesystem hin [4, 25, 26].

Ausbruchssettings sind durch die Gesundheitsämter teilweise schwer zu erheben. Vollständigkeit und Qualität hängen zum einen von verfügbaren Kapazitäten für Ermittlungstätigkeiten vor Ort ab. Zum anderen ist es für Erkrankte aufgrund der zum Teil eher unspezifischen Symptomatik und eines möglicherweise schleichenden Beginns der Erkrankung nicht immer möglich, im Nachhinein den wahrscheinlichen Zeitpunkt der Infektion anzugeben [8]. Es kann nicht ausgeschlossen werden, dass insbesondere leicht zu ermittelnde Settings, wie bspw. der private Haushalt, aufgrund besserer Nachverfolgbarkeit häufiger ermittelt wurden. Bei der Erfassung in der Meldesoftware kann zudem nur zwischen vorgegebenen Kategorien ausgewählt werden. So fallen z. B. Ausbrüche in Fleisch verarbeitenden Betrieben unter das Setting Arbeitsplatz [8].

Im Rahmen der syndromischen Krankenhaus-Surveillance wurde eine etablierte SARI-Falldefinition durch ICD-10-Codes für eine laborbestätigte COVID-19-Erkrankung ergänzt [5, 6]. Zwar wurden im Frühling 2020 COVID-19-Fälle möglicherweise noch untererfasst. Aufgrund der bereits zu Beginn insgesamt gezielten Testung von schweren Fällen mit respiratorischer Symptomatik und des ab Juli systematischen Screenings von Neuaufnahmen in den ICOSARI-Krankenhäusern ist hier 
jedoch von einer eher guten Erfassung in ICOSARI auszugehen. Der Fokus auf ausschließlich COVID-19-Fälle mit einer schweren respiratorischen Symptomatik lässt zwar keine Aussagen zu Fällen ohne respiratorische Symptomatik zu. Im Gegensatz zu Meldungen gemäß IfSG erfolgt die Erfassung in ICOSARI jedoch unabhängig von der bundesweiten Teststrategie [2] und deckt sich daher gut mit den Ergebnissen anderer Analysen zu hospitalisierten COVID-19-Fällen und schweren Verläufen in Deutschland [28, 33, 38]. Darüber hinaus kann es jedoch bei hoher regionaler Belastung des Gesundheitssystems - insbesondere während der Phase 3 - durch die Festlegung von Schwerpunktkliniken und innerdeutschen Verlegungen (Kleeblattkonzept) zu einer Verzerrung der Anzahl an COVID-19-Patienten im ICOSARISystem gekommen sein [31].

Mit dem Abklingen der zweiten Welle zu Beginn des Jahres 2021 stieg der Anteil der besorgniserregenden Virusvariante (Variant of Concern, VOC) B.1.1.7 in Deutschland, lag aber bis zur KW 8/2021 noch unter $50 \%$ [39]. Es ist davon auszugehen, dass der Einfluss der VOC im Jahr 2020 eine untergeordnete Rolle spielte. Nicht ganz ausgeschlossen werden kann aber ein Einfluss auf einzelne Stadt- und Landkreise in Sachsen, die in Phase 3 eine hohe Inzidenz aufwiesen. Diese lagen vor allem an der Grenze zur Tschechischen Republik, in der am Ende das Jahres 2020 bereits eine erhöhte Zirkulation von VOC (insbesondere B.1.1.7) vermutet wurde.

\section{Fazit}

Die COVID-19-Pandemie in Deutschland war bis März 2021 durch verschiedene Phasen geprägt, die durch 2 unterschiedlich schwer verlaufende Wellen im Frühjahr und Herbst und eine Sommerplateauphase mit eher milden Verläufen charakterisiert waren. Im Vergleich zur ersten Welle erstreckte sich die zweite Welle über einen längeren Zeitraum, in dem wöchentlich deutlich mehr COVID-19-Fälle und COVIDSARI-Fälle in den verschiedenen Surveillance-Systemen beobachtet wurden. Mit Blick auf die Altersverteilung wurden in Phasen mit geringerer SARS-CoV-
2-Aktivität (Phase sporadischer Fälle und Sommerplateau) eher jüngere und reiseassoziierte Fälle beobachtet, während dagegen in den beiden COVID19-Wellen auch viele ältere Menschen erkrankten. Unabhängig von den Phasen waren jedoch vor allem ältere Menschen und im Verhältnis mehr Männer als Frauen von schweren bzw. kritischen Krankheitsverläufen betroffen.

Zusätzlich konnte gezeigt werden, dass sich die Meldedaten gemäß IfSG und die syndromische Surveillance gegenseitig ergänzen und auf diese Weise einen guten Überblick zum Gesamtgeschehen in Deutschland hinsichtlich Transmission und Krankheitsschwere geben können.

\section{Korrespondenzadresse}

\section{Julia Schilling}

Abteilung für Infektionsepidemiologie, Robert Koch-Institut

Seestr. 10, 13353 Berlin, Deutschland Schillingj@rki.de

Danksagung. Ein großer Dank gilt den Gesundheitsämtern, der meldenden Ärzteschaft, den Kliniken und den zuständigen Landesgesundheitsbehörden, die seit über einem Jahr neben der lokalen Krisenbewältigung diese wichtigen Informationen zur Bewertung des gesamten Infektionsgeschehens erheben, lokal validieren und an das Robert KochInstitut übermitteln.

Funding. Open Access funding enabled and organized by Projekt DEAL.

\section{Einhaltung ethischer Richtlinien}

Interessenkonflikt. J. Schilling, K. Tolksdorf, A. Marquis, M. Faber, T. Pfoch, S. Buda, W. Haas, E. Schuler, D. Altmann, U. Grote und M. Diercke geben an, dass kein Interessenkonflikt besteht.

Für diesen Beitrag wurden von den Autoren keine Studien an Menschen oder Tieren durchgeführt. Für die aufgeführten Studien gelten die jeweils dort angegebenen ethischen Richtlinien.

Open Access. Dieser Artikel wird unter der Creative Commons Namensnennung 4.0 International Lizenz veröffentlicht, welche die Nutzung, Vervielfältigung, Bearbeitung, Verbreitung und Wiedergabe in jeglichem Medium und Format erlaubt, sofern Sie den/die ursprünglichen Autor(en) und die Quelle ordnungsgemäß nennen, einen Link zur Creative Commons Lizenz beifügen und angeben, ob Änderungen vorgenommen wurden.

Die in diesem Artikel enthaltenen Bilder und sonstiges Drittmaterial unterliegen ebenfalls der genannten Creative Commons Lizenz, sofern sich aus der Abbil- dungslegende nichts anderes ergibt. Sofern das betreffende Material nicht unter der genannten Creative Commons Lizenz steht und die betreffende Handlung nicht nach gesetzlichen Vorschriften erlaubt ist, ist für die oben aufgeführten Weiterverwendungen des Materials die Einwilligung des jeweiligen Rechteinhabers einzuholen.

Weitere Details zur Lizenz entnehmen Sie bitte der Lizenzinformation auf http://creativecommons.org/ licenses/by/4.0/deed.de.

\section{Literatur}

1. Bayerisches Landesamt für Gesundheit und Lebensmittelsicherheit, Robert Koch-Institut (2020) Beschreibung des bisherigen Ausbruchsgeschehens mit dem neuartigen Coronavirus SARS-CoV-2 in Deutschland. EpidBull 2020(7):3-4

2. Schilling J, Buda S, Fischer M et al (2021) Retrospektive Phaseneinteilung der COVID-19Pandemie in Deutschland bis Februar 2021. EpidBull 2021(15):3-12

3. Robert Koch-Institut (2020) Coronavirus-Krankheit-2019. Falldefinition. https://www.rki.de/ DE/Content/InfAZ/N/Neuartiges_Coronavirus/ Falldefinition.pdf.Zugegriffen: 21. Mai 2021

4. Schilling J, Lehfeld A-S, Schumacher D et al (2020) Krankheitsschwere der ersten COVID-19-Welle in Deutschland basierend auf den Meldungen gemäß Infektionsschutzgesetz. J Health Monit S11(5):2-20

5. Buda S, Tolksdorf K, Schuler E et al (2017) Establishing an ICD-10 code based SARI-surveillance in Germany-Description of the system and first results from five recent influenza seasons. BMC Public Health 17(1):612

6. Goerlitz L, Tolksdorf K, Buchholz U et al (2021) Überwachung von COVID-19 durch Erweiterung der etablierten Surveillance für Atemwegsinfektionen. Bundesgesundheitsbl64:395-402.https:// doi.org/10.1007/s00103-021-03303-2

7. Robert Koch-Institut (2020) Leitfaden für den Öffentlichen Gesundheitsdienst zum Vorgehen bei Häufungen von COVID. Robert Koch-Institut, Berlin. https://www.rki.de/DE/Content/InfAZ/ N/Neuartiges_Coronavirus/Leitfaden_OEGD_ COVID-19.pdf.Zugegriffen:20. März 2021

8. Buda S, An der Heiden M, Altmann D et al (2020) Infektionsumfeld von erfassten COVID-19Ausbrüchen in Deutschland. EpidBull 38:3-12

9. Destatis (2019) Grunddaten der Krankenhäuser. Gesundheit Fachserie 12 (Reihe 6.1.1). Statistisches Bundesamt. https://www.destatis.de/ DE/Themen/Gesellschaft-Umwelt/Gesundheit/ Krankenhaeuser/Publikationen/DownloadsKrankenhaeuser/grunddaten-krankenhaeuser2120611197004.pdf.Zugegriffen: 23. März 2021

10. World Health Organization (2020) Report of the WHO-China joint mission on Coronavirus disease 2019 (COVID-19). World Health Organization (WHO), Geneva

11. Schilling J, Diercke M, Altmann D et al (2020) Vorläufige Bewertung der Krankheitsschwere von COVID-19 in Deutschland basierend auf übermittelten Fällen gemäß Infektionsschutzgesetz. EpidBull 2020(17):3-9

12. Destatis (2020) Fortschreibung des Bevölkerungsstandes. Statistisches Bundesamt, Wiesbaden. https://www.destatis.de/DE/Methoden/ Qualitaet/Qualitaetsberichte/Bevoelkerung/ 
bevoelkerungsfortschreibung-2019.pdf. Zugegriffen: 30. März 2021

13. Böhmer MM, Buchholz U, Corman VM et al (2020) Investigation of a COVID-19 outbreak in Germany resulting from a single travel-associated primary case: a case series. Lancet Infect Dis 20(8):920-928

14. Alpers K, Haller S, Buchholz U et al (2021) Untersuchung von SARS-CoV-2-Ausbrüchen in Deutschland durch Feldteams des Robert KochInstituts, Februar-Oktober 2020. Bundesgesundheitsbl 64:446-453. https://doi.org/10.1007/ s00103-021-03296-y

15. Robert Koch-Institut (2020) Situationsbericht vom 04.03.2020. Robert Koch-Institut, Berlin. https:// www.rki.de/DE/Content/InfAZ/N/Neuartiges_ Coronavirus/Situationsberichte/2020-03-04-de. pdf.Zugegriffen:23. März 2021

16. Böhm S, Woudenberg T, Chen D et al (2021) Epidemiology and transmission characteristics of early COVID-19 cases, 20 January-19 March 2020, in Bavaria, Germany. Epidemiol Infect 149:e65

17. Correa-Martínez CL, Kampmeier $S$, Kümpers $P$ et al (2020) A pandemic in times of global tourism: superspreading and exportation of COVID-19 cases from a ski area in Austria. J Clin Microbiol 58(6):e588-520

18. Laufs J, Elsbernd H, Holzl F et al (2020) Coronavirus (SARS-CoV-2) - Outbreak in North Rhine-Westphalia (district of Heinsberg)-First diagnosis of a COVID-19 manifestation in a couple. Dtsch Med Wochenschr 145(10):665-669

19. Brandl M, Selb R, Seidl-Pillmeier S et al (2021) Kontrolle eines COVID-19-Ausbruches im Landkreis Tirschenreuth, März bis Mai 2020. EpidBull 2021(12):3-12

20. Robert Koch-Institut (2020) Situationsbericht vom 19.03.2020. Robert Koch-Institut, Berlin. https:// www.rki.de/DE/Content/InfAZ/N/Neuartiges Coronavirus/Situationsberichte/2020-03-18-de pdf.Zugegriffen:20. März 2021

21. Bund, Länder (2020) MPK-Beschluss vom 27. August 2020

22. Frank C, Hellenbrand W, Sievers C (2021) Betrachtung der reiseassoziierten COVID-19-Fälle im Sommer 2020 unter Berücksichtigung der Schulferien, Reisetätigkeit und Testkapazitäten. EpidBull 2021(8):3-16

23. Robert Koch-Institut (2020) Situationsbericht vom 24.06.2020. Robert Koch-Institut, Berlin. https:// www.rki.de/DE/Content/InfAZ/N/Neuartiges Coronavirus/Situationsberichte/2020-06-24-de. pdf.Zugegriffen:20. März 2021

24. Robert Koch-Institut (2020) Situationsbericht vom 29.07.2020. Robert Koch-Institut, Berlin. https:// www.rki.de/DE/Content/InfAZ/N/Neuartiges Coronavirus/Situationsberichte/2020-07-29-de pdf.Zugegriffen: 20. März 2021

25. Rexroth U, Hamouda O, Hanefeld J, Ruehe B, Wieler LH, Schaade L (2020) Letter to the editor: Wide indication for SARS-CoV-2-testingallowed identification of international risk areas during the early phase of the COVID-19 pandemic in Germany. Euro Surveill. https://doi.org/10.2807/1560-7917. ES.2020.25.23.2001119

26. Stern D, Böttcher S, OhDY etal (2020) Erfassung der SARS-CoV-2-Testzahlen in Deutschland. EpidBull 2021(6):13-19

27. James N, Menzies M (2020) COVID-19 second wave mortality in Europe and the United States. Chaos 30(9):91102

28. Karagiannidis C, Windisch W, McAuley DF et al (2020) Major differences in ICU admissions during the first and second COVID-19 wave in Germany.
Lancet Respir Med 8(9):853-862. https://doi.org/ 10.1016/S2213-2600(21)00101-6

29. Kluge S, Janssens U, Spinner CD et al (2021) Recommendations on inpatient treatment of patient with COVID-19. Dtsch Arztebl Int 118(1-2):1-7

30. Kluge S, Janssens U, Welte Tetal (2021) S3-Leitlinie - Empfehlungen zur stationären Therapie von Patienten mit COVID-1. AWMF, online, Bd. 113/001

31. Gräsner J-T, Hannappel L, Zill Met al (2020) COVID19-Intensivpatienten: Innerdeutsche Verlegungen. Dtsch Arztebl 117(48):4

32. Perumal N, Steffen A, Ullrich A et al (2021) Effekte derCOVID-19-Impfung auf Inzidenzund Todesfälle in der älteren Bevölkerung in Deutschland: erste Ergebnisse einer ökologischen Analyse. EpidBull 2021(19):37-51

33. Tolksdorf K, Buda S, Schuler E et al (2020) Eine höhere Letatlität und lange Beatmungsdauer unterscheiden COVID-19von schwer verlaufenden Atemwegsinfektionen in Grippewellen. EpidBull 41:3-10

34. loannidis JPA, Axfors C, Contopoulos-loannidis DG (2021) Second versus first wave of COVID-19 deaths: shifts in age distribution and in nursing home fatalities. Environ Res 195:110856

35. Williamson EJ, Walker AJ, Bhaskaran K et al (2020) Factors associated with COVID-19-related death using OpenSAFELY. Nature 584(7821):430-436

36. Robert Koch-Institut (2021) Epidemiologischer Steckbrief zu SARS-CoV-2 und COVID-19. https:// www.rki.de/DE/Content/InfAZ/N/Neuartiges_ Coronavirus/Steckbrief.html. Zugegriffen: 20. März 2021

37. Wirth R, Becker C, Djukic M et al (2021) COVID-19 in old age-The geriatric perspective. Z Gerontol Geriatr 54(2):152-160

38. Karagiannidis C, Mostert C, Hentschker $C$ et al (2020) Case characteristics, resource use, and outcomes of 10.021 patients with COVID-19 admitted to 920 German hospitals: an observational study. Lancet Respir Med 8(9):853-862

39. RKI (2021) Bericht zu Virusvarianten von SARSCoV-2 in Deutschland, Stand 12. Mai 2021. Robert Koch-Institut, Berlin. https://www.rki.de/ DE/Content/InfAZ/N/Neuartiges_Coronavirus/ DESH/Bericht_VOC_2021-05-12.pdf.Zugegriffen: 21. Mai 2021

40. World Health Organization (2020) Timeline of WHO's response to COVID-19. https:// www.who.int/news-room/detail/29-06-2020covidtimeline.Zugegriffen:27. Mai 2021

41. BMI/BMG GK (2020) Pressemitteilung. Absage aller Veranstaltungen ab 1.000 Teilnehmern. https:// www.bundesgesundheitsministerium.de/presse/ pressemitteilungen/2020/1-quartal/krisenstabbmg-bmi-sitzung-5.html. Zugegriffen: 13. März 2021

42. Tagesschau.de (2020) Wo bleiben Schulen zu und wie lange? Norddeutscher Rundfunk, Hamburg. https://www.tagesschau.de/inland/coronaschulschliessungen-103.html. Zugegriffen: 29 Juni 2021

43. MPK (2020) MPK-Beschluss vom 22.03.2020 Ministerpräsidentenkonferenz. https://www. bmi.bund.de/SharedDocs/downloads/DE/ veroeffentlichungen/2020/corona/hinweiseinschraenkung-soziale-kontakte.pdf. Zugegriffen: 13. März 2021

44. BMI (2020) Pressemittelung vom 09.04.2020. Bund und Länder haben sich auf einheitliche Quarantäneregeln für Einreisende verständigt. https://www.bmi.bund.de/SharedDocs/ pressemitteilungen/DE/2020/04/muster- verordnung.html. Zugegriffen: 13. März 2021

45. MPK (2020) MPK-Beschluss vom 15.04.2020. Ministerpräsidentenkonferenz (Stand: 13.03.2021)

46. KMK (2020) Beschluss der KMK vom 28.04.2020. Rahmenkonzept zur Wiederaufname von Unterricht an Schulen, Deutschland

47. Bundesregierung (2020) Beschlüsse von Bund und Ländern (17.04.2021). https:// www.bundesregierung.de/breg-de/themen/ coronavirus/bund-laender-corona-1744306. Zugegriffen: 13. März 2021

48. MPK (2020) MPK-Beschluss vom 30.04.2020. Ministerpräsidentenkonferenz. https://lexcorona. de/lib/exe/fetch.php?media=rechtsaktebund: 2020-04-30-beschluss-bund-laender-data.pdf. Zugegriffen: 13. März 2021

49. MPK (2020) MPK-Beschluss vom 06.05.2020. Ministerpräsidentenkonferenz. https://www. bundesregierung.de/resource/blob/973812/ 1750978/fc61b6eb1fc1d398d66cfea79b565129/ 2020-05-06-beschluss-mpk-data.pdf. Zugegriffen: 13. März 2021

50. Verordnung zur Änderung der Verordnung zum Anspruch auf bestimmte Testungen für den Nachweis des Vorliegens einer Infektion mit dem Coronavirus SARS-Cov-2 vom 31.07.2020, Deutschland

51. deutschland.de (2021) Die Bundesregierung informiert über die Corona-Krise. https://www. deutschland.de/de/news/bundesregierung-undcorona-krise.Zugegriffen: 13. März 2021

52. Auswärtiges Amt (2020) Länderspezifische Reisewarnungen aufgrund der COVID-19-Pandemie. https://www.auswaertiges-amt.de/de/ ReiseUndSicherheit/covid-19/2296762. Zugegriffen: 15. März 2021

53. MPK (2020) MPK-Beschluss vom 14. Oktober 2020 Ministerpräsidentenkonferenz. https://www. bundesregierung.de/resource/blob/997532/ 1798920/9448da53f1fa442c24c37abc8b0b2048/ 2020-10-14-beschluss-mpk-data.pdf. Zugegriffen: 13. März 2021

54. Bundesregierung (2020) Videokonferenz der Bundeskanzlerin mit den Regierungschefinnen und Regierungschefs der Länder am 28. Oktober 2020.https://www.bundesregierung.de/breg-de/ themen/buerokratieabbau/videokonferenz-derbundeskanzlerin-mit-den-regierungschefinnenund-regierungschefs-der-laender-am-28oktober-2020-1805248. Zugegriffen: 13. März 2021

55. MPK (2020) MPK-Beschluss 25. November 2020 Ministerpräsidentenkonferenz. https://www. bundesregierung.de/resource/blob/975232/ 1820090/11c9749f77a71b9439759538864aa672/ 2020-11-25-mpk-beschluss-data.pdf. Zugegriffen: 13. März 2021

56. MPK (2020) MPK-Beschluss 13. Dezember 2020 Ministerpräsidentenkonferenz. https://www. bundesregierung.de/resource/blob/975226/ 1827366/69441fb68435a7199b3d3a89bff2c0e6/ 2020-12-13-beschluss-mpk-data.pdf. Zugegriffen: 13. März 2021

57. MPK (2021) MPK-Beschluss vom 05. Januar 2021. Ministerpräsidentenkonferenz. https://www. bundesregierung.de/resource/blob/975226/ 1834306/75346aa9bba1050fec8025b18a4bb1a3/ 2021-01-05-beschluss-mpk-data.pdf. Zugegriffen: 27. Mai 2021

58. Faria NR, Claro IM, Candido D et al (2021) Genomic characterisation of an emergent SARSCoV-2 lineage in Manaus: preliminary findings. virological.org, Sao Paulo, Brasilien. https:// 


\section{Leitthema}

virological.org/t/genomic-characterisation- ofan-emergent-sars-cov-2-lineage-in-manauspreliminary-findings/586. Zugegriffen: 29. Juni 2021

59. MPK (2021) MPK-Beschluss vom 19. Januar 2021. Ministerpräsidentenkonferenz. https://www. bundesregierung.de/resource/blob/997532/ 1840868/1c68fcd2008b53cf12691162bf20626f/ 2021-01-19-mpk-data.pdf. Zugegriffen: 27. Mai 2021

60. BMG (2021) Neue Coronavirus-Surveillanceverordnung des Bundesministeriums für Gesundheit Bundesministerium fürGesundheit, Berlin.https:// www.bundesgesundheitsministerium.de/presse/ pressemitteilungen/2021/1-quartal/coronavirussurveillanceverordnung.html.Zugegriffen:27. Mai 2021

61. MPK (2021) MPK-Beschluss vom 10. Februar 2021. Ministerpräsidentenkonferenz. https://www. bundesregierung.de/resource/blob/997532/ 1852514/508d851535b4a599c27cf320d8ab69e0/ 2021-02-10-mpk-data.pdf. Zugegriffen: 27. Mai 2021 